Reflective testing

\section{Reflective testing}

\section{W G Simpson, P J Twomey}

\section{Providing added value}

$\mathrm{T}$ he processing of chemical pathology requests includes pre-analytical, analytical, and post-analytical phases. At its most basic, the postanalytical process is the reporting and delivery of validated results. One of the ways in which the chemical pathology laboratory provides "added value" over a purely analytical service is at this postanalytical stage- that is, further processing of results related to the requesteither before issuing the report, simultaneously, or at a later stage. Indeed, such post-analytical processing is integral to the provision of a quality service, and as such is a requirement for laboratory accreditation in the UK. ${ }^{1}$

The simplest form of processing is to compare the result with an appropriate, predefined "action range" (range checking), or to compare changes in sequential results from the same individual to predefined expected ranges of change (delta checking). Historically, this would have been carried out visually by the person authorising paper reports, but now such checks are usually performed by laboratory information systems, which automatically authorise results and generate reports for results that do not fail the checks. Although in many cases the results that fail these tests will be deemed to be acceptable in the clinical circumstances and so no further action would be required, others will require action. This may be as simple as telephoning unexpected results to facilitate earlier intervention, but processing can involve adding comments or requesting appropriate further tests on the sample (reflex testing). Some laboratory information systems will perform these actions automatically but, although expert systems are becoming available, ${ }^{2}$ such automated processing currently remains fairly basic.

In this issue, Paterson and Paterson examine an alternative form of processing, for which they use the term "reflective testing". ${ }^{3}$ This is the process by which a laboratory clinician might add on further tests using their clinical judgement. Although this is common practice, the process does not appear to have been evaluated previously, and deserves further scrutiny, particularly because different individuals take dif-

ferent approaches. The question of best practice with regard to adding on of tests has been the matter of some debate. The authors make some assessment of the clinical effectiveness of their strategy, although cost effectiveness and ethical issues also have to be considered.

With regard to clinical effectiveness, the authors use the term NND-the number of add on tests that are required to obtain a specific diagnosis. This would seem a logical way to compare the effectiveness of different strategies of investigation, based simply on the proportion of add on tests that yield definitive results, although the sensitivity and specificity (and thus negative and positive predictive values) of the approach are not fully examined. The quality of clinical details would have a major influence on NND-the more information, the more added value can be provided. Furthermore, variability of clinical insight and experience will also have an influence, hence NND would vary between individuals.

\section{"There seems to be no formal advice for chemical pathologists to cover the ethics of add on tests, and there is a need for professional bodies to examine this whole area"}

Cost effectiveness is potentially more complex to evaluate. At its simplest, one might take just the basic laboratory costs into account-the analytical costs in addition to the personnel time (which would be considerably higher for reflective rather than automated reflex testing). More difficult to measure would be the overhead incurred through disruption of the workflow in the modern laboratory, because this often necessitates manual intervention by biomedical scientific staff. There will be costs, to both the patient and the health service, incurred by finding the diagnosis and the potential treatment thereafter. There may, indeed, be costs of missing a diagnosis, or, moreover, what would be the cost of an incorrect diagnosis resulting from an added on test (both to society and the patient)? It is difficult to assess the difference between reflex and reflective testing in these regards, and there is a need for "evidence based outcomes analysis". ${ }^{4}$

These questions would have to be addressed for each strategy. The ethical questions are perhaps more general, but certainly a matter of great debate. ${ }^{5}$ Samples and related requests are sent to the laboratory with the patient's implied consent; is it ethical therefore to perform a test on a sample without the patient's consent, or should samples be viewed as "referrals", whereby not performing an appropriate, more detailed, examination could be deemed to be negligent? Is the test needed to fulfil your specialist contribution to patient care? Is it superfluous to management of the presenting problem? It has been suggested that, if the result of the test would be in the interests of the patient, then it would be ethical to add it on (EM Russell, personal communication, 2003). Above all, no harm (in the broadest sense) should come to the patient from adding on a test. Unfortunately, there seems to be no formal advice for chemical pathologists to cover the ethics of add on tests, and there is a need for professional bodies to examine this whole area.

Although few clinicians would argue with laboratory generated additional requesting of a thyroid hormone estimation if a thyroid stimulating hormone result is abnormal, there is considerable debate about the ethics of adding on electrophoresis when a high protein is found. In some respects, reflex testing can circumvent the ethical issue. Because such strategies per se have to be designed in advance, it should be possible to agree with requesters, in advance, that "in situation $\mathrm{X}$, the laboratory will routinely undertake extra tests $\mathrm{Y}^{\prime \prime}$. However, in such agreed strategies, it may still be debated whether a patient has given "informed" consent to the addition of the extra test $\mathrm{Y}$ if the consequences of the test have not been explained. Reflective testing is, by definition, more subjective (although it should be emphasised that the threshold values used in reflex testing are arbitrarily chosen without an evidence base).

Clinical governance has correctly placed an emphasis on clinical quality. However, there has been little research on post-analytical quality in chemical pathology. Paterson and Paterson have made an important step on the road to defining post-analytical quality. As they have correctly stated, there is a need for more research in the area and hopefully their paper will stimulate this work. Is there also a case to be made for reflective deletion of test requests?

J Clin Pathol 2004;57:239-240.

doi: 10.1136/jcp.2003.011668 


\author{
Authors' affiliations \\ W G Simpson, Department of Clinical \\ Biochemistry, Aberdeen Royal Infirmary, \\ Foresterhill, Aberdeen AB25 2ZD, UK \\ P J Twomey, Department of Clinical \\ Biochemistry, Edinburgh Royal Infirmary, \\ Edinburgh EH16 4SA, UK \\ Correspondence to: $\operatorname{Dr}$ W G Simpson, \\ Department of Clinical Biochemistry, Áberdeen \\ Royal Infirmary, Foresterhill, Aberdeen AB25 \\ 2ZD, UK;
}

w.g.simpson@arh.grampian.scot.nhs.uk

Accepted 12 December 2003

\section{REFERENCES}

1 Clinical Pathology Accreditation (UK) Ltd http://www.cpa-uk.co.uk (last accessed 06/12/ 03).

2 Smith BJ, McNeely MDD. The influence of an expert system for test ordering and interpretation on laboratory investigations. Clin Chem 1999;45: $1168-75$.
3 Paterson JR, Paterson R. Reflective testing: how useful is the practice of adding on tests by laboratory clinicians? J Clin Pathol 2004;57:273-5.

4 Wu AHB. Improving the utilization of clinical laboratory tests. J Eval Clin Pract 1997:4:171-81.

5 Challand G. Ethics of add-on tests. ACB discussion forum posting, 25/09/2002. http:// www.jiscmail.ac.uk/cgi-bin/wa.exe?A2 = ind $0209 \& \mathrm{~L}=$ acb-clin-chem-gen \&D $=0 \& \mathrm{I}=$ $-1 \& T=0 \& P=10974$ (last accessed 06/12/03).

6 Rao GG, Crook M, Tillyer ML. Pathology tests: is the time for demand management ripe at last? $J$ Clin Pathol 2003;56:243-8.

\section{$\mathrm{ECHO}$}

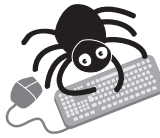

Please visit the Journal of Clinical Pathology website [www. jclinpath.com] tor a link to the full text of this article.

\section{Gene expression profiles in gastrointestinal stromal tumours}

t

nitial clinical features and histopathology often fail to distinguish between benign and malignant gastrointestinal stromal tumours (GISTs). These tumours probably originate in the intestinal stem cells of Cajal and it is not possible to compare gene expression profiles of the malignant tumours and the normal stem cells. Researchers in Virginia, USA have compared benign with malignant tumours.

They studied 25 GISTs, all c-KIT-positive on immunohistochemistry and with GIST histology. At diagnosis they were classified as benign (8), malignant (11), or uncertain (6). In gene array analyses performed on the first nine tumours ( 3 benign, 6 malignant) the expression of 1174 genes was evaluated for each sample. Compared with benign GISTs, malignant GISTs showed overexpression of 27 genes of which eight were overexpressed in five or more of the six malignant tumours. The eight genes were VIL2, COL8Al, CCNBI, HMG2, TSG101 tumour susceptibility protein, CENP-F kinetochore protein, protein tyrosine kinase 2 (FAK), and protein kinase DYRK2. Quantitative real time RT-PCR confirmed these findings. When the additional 16 tumours were assessed using quantitative real time RTPCR the five malignant tumours showed similar gene overexpression. Three of the six patients whose tumours were originally classified as of uncertain malignant potential died of the disease and their tumours segregated with the other malignant tumours as regards gene expression. The tumours of the three patients in this group who had no evidence of malignancy on follow up showed the benign gene expression profile. The six genes most significantly overexpressed in malignant tumours were VIL2, CCNB1, HMG2, TSG101, CENP-F, and FAK. The two gene pairs most significantly overexpressed in malignant tumours were CCNBI-CENP-F and CCNBI-FAK.

These findings help in understanding the oncogenesis of GISTs and provide a means of distinguishing between benign and malignant tumours.

A Gut 2004;53:235-240. 